\title{
Serial structures as response items in paired-associate learning
}

\author{
L. BERELL KORNREICH, ANDREW \\ $K A N E$, and JOSEPH STRAKA, University \\ of Wisconsin, Milwaukee, Wis. 53201
}

In three different experiments, 16 names were paired with four response items which represented a serial structure. The items were " $H . S$. diploma," "B. A.," "M. A.," and "Ph.D." for Experiment 1; "city," "county," "state," and "country" for Experiment 2; and "city," "county," "country," and "continent" for Experiment 3. Results for each experiment showed that the end items were learned faster in terms of trials to a criterion and errors before criterion. Forward association errors were highly related to corresponding backward association errors for Experiments 2 and 3. In the same two experiments errors involving "county" and "country" were markedly different, which was interpreted as further evidence of the effect of a previously learned serial structure upon paired-associate learning.

Accumulating evidence indicates that if a set of words, which exists as a series in our culture, is used as responses in a pairedassociate learning task, its learned serial structure produces a serial position effect even though the words are presented in a random order (De Soto \& Bosley, 1962; Pollio \& Draper, 1966). These findings indicate that a set of stimuli which were originally learned in a serial order will subsequently produce learning rates and error patterns which reflect a serial structuring in a paired-associate learning task.

The present experiments sought to extend the previous findings using different stimulus and response items. Three different serial structures were used as response items, while the stimulus items remained the same throughout the three experiments. The second experiment provides a test of strength between stimulus similarity effects and serial structure effects. A comparison of the second and third experiment enables one to observe the effect of changing an item's position in the serial structure.

Since all three experiments involved essentially the same procedure, only the specific procedure used in Experiment 1 will be reported in detail. A description of the points of difference will be noted under Experiments 2 and 3.

\section{EXPERIMENT 1 \\ Subjects}

Twenty-seven students in undergraduate psychology courses at the University of Wisconsin-Milwaukee served in this experiment as part of a course requirement. Materials

Sixteen $3 \times 5$ cards, each with a man's first and last name typed on the front, and with one of four response items (H.S. diploma, BA, MA, or PhD) typed on the back, was prepared. The men's names were drawn at random from the local telephone book. Four packs of cards were constructed, so each response item was paired with four different names in each pack. In this way, any effects due to the men's names were counterbalanced.

\section{Procedure}

Each $S$ was tested individually using one of the four packs. A criterion of two successive trials through the entire pack without an error was employed, and the 16 cards were shuffled between each trial in order to vary the order of presentation. Each $\mathrm{S}$ was read the same instructions (similar to those used by DeSoto \& Bosley, 1962) and told to learn the label, "H.S. diploma," "BA," "MA," or "PhD," which went with each name by looking at the name and then turning the card over to see the label. They were told to spend no more than a few seconds per card. After the first trial, $S$ was required to give an answer for each card.

\section{Results and Discussion}

The effect due to the pack used was examined by an analysis of variance and was not significant $(F<1.0)$. Therefore, the remaining analyses were based on data from all Ss without regard to the stimulus pack they received. The data based on the number of trials required to meet the criterion will be considered first. The number of trials each $S$ took to anticipate correctly all 16 responses twice in succession was computed for each response item and averaged across all Ss. The results were as follows: "H.S. diploma" = $11.7 ;$ "BA" = 13.1; "MA" = 12.6; and "PhD" = 10.5. An analysis of variance for these data was highly significant $(F=218, p<.01)$. NewmanKeuls tests indicated that all comparisons, except for "MA" and "BA,"were significant at beyond the .01 level. Thus, in terms of trials to a criterion, the middle items in the serial structure were significantly more difficult to learn that the end items. The data from Experiment 1 for errors made before the criterion was met are presented in Fig. 1. Each curve is a plot of the 12 possible error types for that experiment. These error data again indicate that the middle items are most troublesome to learn, even though they are guessed at least as frequently as the end items. Also by far the most confusions involve the two middle items. That is, BA is called MA and vice versa more often than other types of errors.

Experiment 1 suggested that the serial structure of the response items did influence the learning rate and type of errors made in the paired-associate task, even though individual paired associates were not presented in a serial order. However, it could be argued that Experiment 1 does not provide a strong test of the serial structure hypothesis, since the physical similarity of the middle items might have caused both the trials to criterion and the type of errors effects. In other words, "BA" and "MA" both contain As, and so may have presented difficulty due to stimulus generalization. Experiment 2 was run in order to permit a stronger test of the serial structure hypothesis.

\section{EXPERIMENT 2 Subjects}

Twenty-six students in undergraduate psychology courses at the University of Wisconsin-Milwaukee served in this experiment as part of a course requirement. Materials

The materials were identical to those used in Experiment 1 except that the response items differed. The words "city," "county," "state," and "country" served as the response items. These words represent an interesting serial structure because "county" and "country" are physically identical except for the " $r$ " in "country," but are two steps apart in the serial structure. The words "county" and "state" are very dissimilar physically but are adjacent in the serial structure. The stimulus generalization hypothesis predicts more confusions between "county" and "country," whereas the serial structure hypothesis predicts more confusions between "county" and "state."

Procedure

There were no differences in procedure between Experiment 1 and Experiment 2.

Results and Discussion

The effect due to the pack used was again not significant $(F=1.8, p>.10)$. Therefore, the remaining analyses disregarded the "pack" variable. The trials-to-criterion results were: "city" = 15.7; "county" = 16.5; "state" = 15.9; and "country" $=13.2$. An analysis of variance indicated that these means differed significantly $(F=3.85 ; p<.05)$. When individual means were compared by the Newman-Keuls test, the city-country and the state-country comparisons were significant beyond the .05 level, and the county-country difference was significant beyond the .01 level. Thus the "country" items were learned significantly 


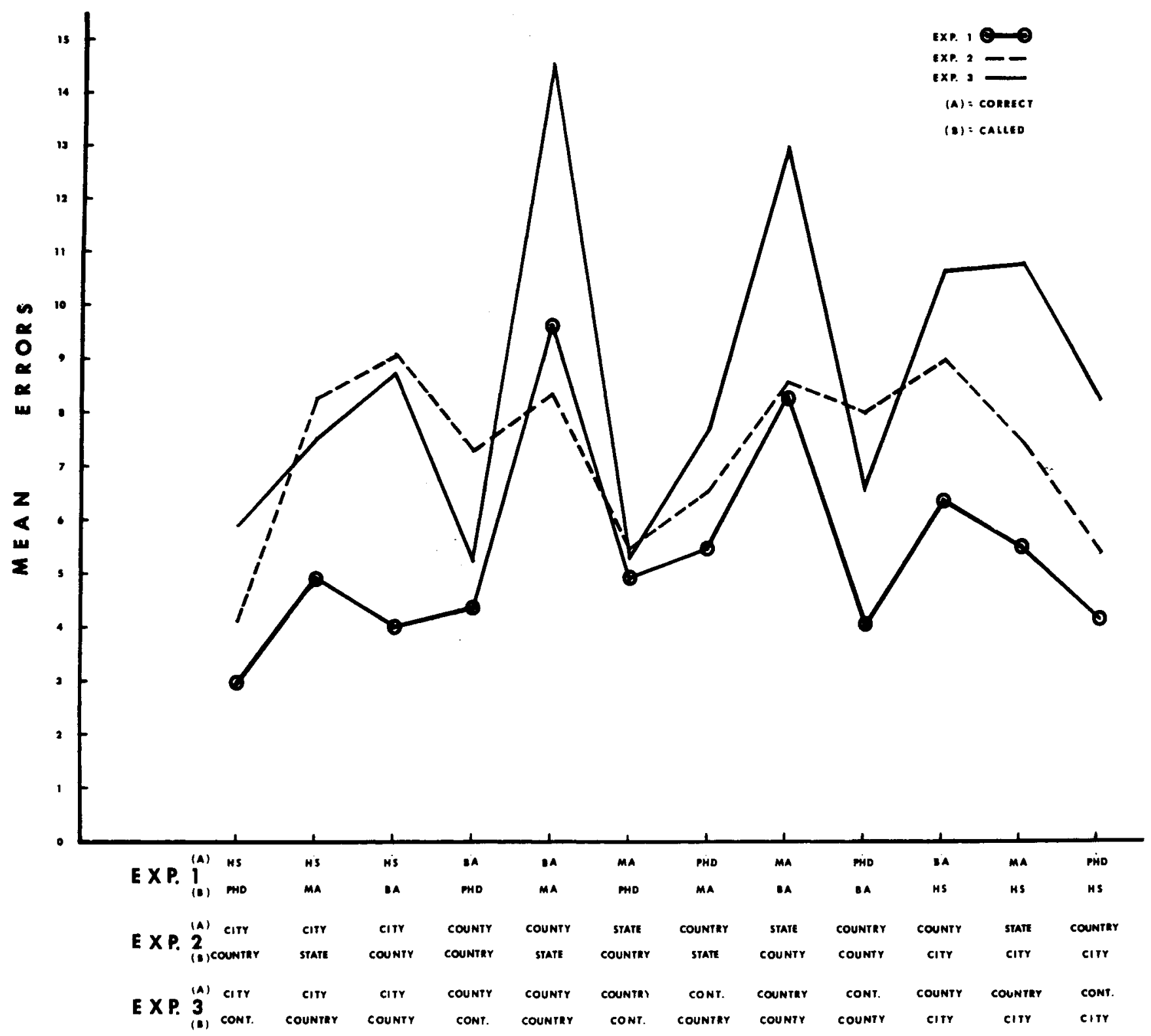

Fig. 1. Mean number of errors for the 12 error types for Experiments 1, 2, and 3.

faster than the other three types, while they did not differ from each other.

The critical data in this experiment are the error data and are presented in Fig. 1. Fewest errors are made on "country." Consistent with the trials-to-criterion data, the errors and guessing tendencies for the other three response items are not markedly different. However, the comparisons of interest involve particular confusions. "County" is called "country" by mistake an average of 7.31 times, whereas "county" is called "state" an average of 8.35 times. In the reverse comparison, "country" is called "county" by mistake an average of 8.08 times, while "country" is called "state" an average of 6.54 times.

These results clearly support neither the stimulus generalization hypothesis nor the serial position hypothesis. Perhaps the fact that there were more confusions between "city" and "county," adjacent items in the serial structure, than between "county" and "country" is supportive of the importance of the serial structure variable. It could be argued that the word "state" introduced a Von Restorff effect, in that it was the only word beginning with "s." In comparing Experiment 2 with Experiment 1, the major differences in results involve "state," both in terms of fewer relative trials to criterion and fewer errors for its relative position in the serial structure. A third experiment was run in order to assess the argument that "state," due to its uniqueness, was learned faster and with fewer errors, thereby masking the effect of a serial structure which was also present. Experiment 3 is also the first example in the literature of varying a single item in a serial structure, in order to observe the stability of the effects of that structure. EXPERIMENT 3

Subjects

Twenty-eight students in undergraduate psychology courses at the University of Wisconsin-Milwaukee served in this experiment as part of a course requirement.

\section{Materials}

The materials were identical to those used in Experiment 2 except that another response item was substituted for "state." The words "city," "county," "country," and "continent" served as the response items. 


\section{Procedure}

There were no differences in procedure between Experiment $3 *$ and either Experiment 2 or Experiment 1.

\section{RESULTS AND DISCUSSION}

Again the effect due to pack was not significant $(F<1)$. The remaining analyses disregarded the pack variable. The data for trials to criterion were: "city" $=14.3$; "county" = 15.9; "country" = 15.9; "continent" =12.3. An analysis of variance indicated significant differences among the means $(F=5.80, p<.01)$. The NewmanKeuls test revealed that the city-continent difference was significant at beyond the .05 level, and the county-continent and the country-continent differences were significant at beyond the .01 level. These results based on trials to criterion very nicely replicate the comparable results from Experiment 2.

One indication of a serial structure which has not yet been considered is the degree of symmetry between forward and backward association errors, which can be seen for all three experiments in Fig. 1. The first six points can be thought of as errors which involve forward associations along the serial structure. The last six points indicate backward association errors. For instance, the first error type on the figure involves calling the last item in the serial structure when the first item is correct, e.g., calling "PhD" when "H.S. diploma" is correct. The last error type on the figure is the inverse, e.g., calling "H.S. diploma" when "PhD" is the correct response.

The more symmetrical the error curve, i.e., the more that each of the six forward association error types occupies the same rank as its corresponding backward association error type in terms of difficulty, the more evidence of a serial structure in operation. In order to test the degree of symmetry in each of the three curves, a Spearman Rho was computed for each one. The six forward association errors were ranked from most to least and correlated with the corresponding six backward associations. For Experiment 1 the Rho was .54 , which is not statistically significant $(.83=\mathrm{p}<.05)$. However, for both Experiment 2 and Experiment 3 the Spearman Rho $=.94 ; p<.01$. These data offer strong evidence that a serial structure is affecting the error patterns, at least in Experiments 2 and 3.

Experiments 2 and 3 can be thought of as different conditions within a single experimental design. The independent variable is the presence of either "state" or "continent" as a response item. In comparing the two conditions, e.g., Experiments 2 and 3 , it can be seen that the major difference involves the county-state and state-county error types in Experiment 2 relative to the corresponding county-country and countrycounty error types in Experiment 3 . The rate of errors sharply increases when "state" is taken out of the serial structure. Thus it is likely that the uniqueness of that item. facilitated its learning, whereas its position in the serial structure represented a difficulty. Thus the two effects summed to create an item of moderate difficulty in Experiment 2. The rest of the curves for the two experiments are remarkably similar, which suggests that the serial structure does provide a stable influence on the error patterns. The fact that identical error types, county-country and country-county, occupy very different positions on the error curves of Experiments 2 and 3 testifies to the influence of the serial structure on error patterns.
The three experiments reported here extend the generality of the phenomena first reported by DeSoto \& Bosley (1962), and also provide supportive evidence for the general theory of serial learning recently proposed by Harcum (1967), which emphasizes the learning strategies and the cognitive structures which $S$ brings to the experimental task.

\section{REFERENCES}

DeSOTO, C. B., \& BOSLEY, J. J. The cognitive structure of a social structure. Joumal Abnormal \& Social Psychology, 1962, 64, 303-307.

HARCUM, R. R. Parallel functions of serial learning and tachistoscopic pattem perception. Psychological Review, 1967, 74, 51-62.

POLLIO, H. R, \& DRAPER, D. O. The effect of a serial structure on paired-associate learning. Journal of Verbal Learning \& Verbal Behavior $1966,5,301-308$.

\section{Rhyme as a determinant of clustering'}

\section{WESTON A. BOUSFIELD and DAVID $A$. WICKLUND, University of Connecticut, Storrs, Conn. 06268}

This study dealt with rhyme as a determinant of clustering in free recall. The stimuli comprised 12 rhyming pairs of words. The results from $30 \mathrm{Ss}$ showed significant clustering as well as high variance attributable to both Ss and word pairs.

This study explores the efficacy of rhyme as a determinant of clustering. Words may be said to rhyme when their terminal sounds are similar. The phonetic similarity of given words has apparently not been considered in studies of clustering. A possihle reason for this is that Es have generally relied on conventional free-associational or on category norms for the selection of stimulus items. The latter may be described as being of the restricted associational type. Alternatively stated, these norms have served for the selection of groups of directly or mediationally associated words. In these terms, taxonomic categories may be regarded as mediators with special properties of effectiveness for determining the sequential emission of their subordinate items in free recall. The associational interpretation has shown appreciable predictive power in spite of what now appears to be its relatively narrow range of application. The compilers and users of associational norms have tended to regard clang or rhyming associations as being somehow aberrant or immature in nature. Thus Nobel(1952), in his Hullian analysis of meaningfulness as measured by continued associations, regarded clang responses as unacceptable. Flavel et al (1958) classed clang associations as immature. The fact that clang responses appear in free-associational forms for children but not for adults (Palermo \& Jenkins, 1964) gives further apparent support for the assumption of their immaturity. This somewhat evaluative attitude has probably contributed to their neglect and the favoring of what may loosely be termed logically relevant associative responses. It is becoming increasingly evident, however, that other types of responses and characteristics of words have significant roles in verbal learning. For example, acoustic attributes of words are important for short-term memory (Adams, 1967) and imagery can no longer be neglected (Paivio et al, 1966). Rhyme may be classed as an acoustic attribute, and the present study was pianied to determine its effects on clustering with admitted ignorance regarding factors determining its salience. An exploratory study in whis 1 Ss were presented a list comprised of five sets of four rhyming words showed that Ss did indeed recall the words in clusters of rhymed words. The dimension of rhyming, however, was completely confounded with formal similarity (i.e., fat, mat, rat, sat). The present study is designed to minimize such factors. 